\section{PiggyMac, a domesticated piggyBac transposase involved in programmed genome rearrangements in the ciliate Paramecium tetraurelia}

\author{
Céline Baudry, 1,2,3,4,7 Sophie Malinsky, ${ }^{5,6,7,9}$ \\ Matthieu Restituito, ${ }^{5}$ Aurélie Kapusta, ${ }^{1,2,3,4}$ \\ Sarah Rosa, ${ }^{1}$ Eric Meyer, ${ }^{5}$ and \\ Mireille Bétermier $1,2,3,4,8$
}

${ }^{1}$ CNRS FRE 3144, Centre de Génétique Moléculaire, Gif-surYvette F-91198 Cedex, France; ${ }^{2}$ Université Paris 11, Département de Biologie, Orsay F-91405, France; ${ }^{3}$ Université Pierre et Marie Curie-Paris 6, UFR des Sciences de la Vie, Paris F-75005, France; ${ }^{4}$ CNRS FRC 3115, Centre de Recherches de Gif-sur-Yvette, Gif-sur-Yvette F-91198 Cedex, France; ${ }^{5}$ CNRS UMR 8541-Ecole Normale Supérieure, Paris F-75005, France; ${ }^{6}$ Université Paris Diderot-Paris7, UFR des Sciences du Vivant, Paris F-75205 Cedex 13, France

Programmed genome rearrangements drive functional gene assembly in ciliates during the development of the somatic macronucleus. The elimination of germline sequences is directed by noncoding RNAs and is initiated by DNA double-strand breaks, but the enzymes responsible for DNA cleavage have not been identified. We show here that PiggyMac (Pgm), a domesticated piggy$B a c$ transposase, is required for these rearrangements in Paramecium tetraurelia. A GFP-Pgm fusion localizes in developing macronuclei, where rearrangements take place, and RNAi-mediated silencing of PGM abolishes DNA cleavage. This is the first in vivo evidence suggesting an essential endonucleolytic function of a domesticated piggyBac transposase.

Supplemental material is available at http://www.genesdev.org. Received July 3, 2009; revised version accepted September 4, 2009.

\section{Results and Discussion}

A hallmark of ciliates is the separation of two versions of the genome-a germline and a rearranged somatic version-into two functionally distinct nuclei, a micronucleus (MIC) and a macronucleus (MAC), respectively, that coexist in the cytoplasm of these unicellular eukaryotes. The MAC is responsible for gene transcription but is lost at each sexual cycle, while the MIC undergoes meiosis

[Keywords: Transposase domestication; IES excision; chromosome fragmentation; oligohymenophorean ciliates]

${ }^{7}$ These authors contributed equally to this work.

Corresponding authors.

${ }^{8}$ E-MAIL mireille.betermier@cgm.cnrs-gif.fr; FAX 33-1-69823181.

'E-MAIL malinsky@biologie.ens.fr; FAX 33-1-44323941.

Article is online at http://www.genesdev.org/cgi/doi/10.1101/gad.547309. and gives rise to new MICs and MACs in sexual progeny. During new MAC development, the genome undergoes massive DNA amplification, together with extensive rearrangements (Jahn and Klobutcher 2002; Yao et al. 2002). In Paramecium, imprecise elimination of germline sequences, including essentially all transposable elements, leads to chromosome fragmentation or to internal deletions (Le Mouël et al. 2003). In addition, the precise excision of $\sim 60,000$ single-copy, noncoding internal eliminated sequences (IESs) is essential for the assembly of functional genes (for review, see Bétermier 2004). IESs are invariably bounded by two $5^{\prime}$-TA-3' dinucleotides, only one of which remains in the MAC sequence after excision, and a loosely conserved 8-base-pair (bp) consensus sequence $\left(5^{\prime}\right.$-TAYAGYNR-3') was defined from a statistical analysis of their ends (Klobutcher and Herrick 1995). This consensus shows some similarity to the ends of Tc/mariner class II transposons, which duplicate their TA target site upon insertion, and it was proposed that Paramecium IESs originate from an ancient invasion of the MIC genome by Tc/mariner elements that would have degenerated during evolution, while remaining under selective pressure for precise excision from the MAC genome. It was suggested that an ancestral Tc/mariner transposase could have been domesticated by the host to catalyze efficient excision of inactivated elements (Klobutcher and Herrick 1997). However, Paramecium IES excision starts with staggered double-strand breaks (DSBs) generating 4-base 5' overhangs at both IES ends, centered on the conserved TA /Gratias and Bétermier 2003). Subsequent pairing of the TA dinucleotides may drive the precise closure of IES excision junctions. This geometry differs significantly from the DNA cleavage that initiates Tc/mariner cut-and-paste transposition, which produces 3 ' overhangs and leaves the two flanking TAs at the donor site (Plasterk and van Luenen 2002).

In contrast, piggyBac class II transposons generate no footprint at their donor site, which is reconstituted precisely upon excision to restore the initial 5'-TTAA-3' target sequence (Elick et al. 1996). In vitro, the piggyBac transposase catalyzes double-strand cleavage at transposon ends, and the resulting DSBs exhibit 4-base $5^{\prime}$ overhangs carrying the duplicated TTAA target site (Mitra et al. 2008). Our attention was drawn to a Paramecium tetraurelia gene, GSPATG00016627001 or PiggyMac $(P G M)$, which encodes a putative 1065 -amino-acid protein with significant homology with a piggyBac transposase (Supplemental Fig. S1). Alignment of the predicted Pgm protein with piggyBac transposases (Fig. 1A; Supplemental Fig. S1) reveals the conservation of the DDD catalytic triad and of a downstream cysteine-rich putative plant homeodomain (PHD) finger (Sarkar et al. 2003; Mitra et al. 2008). Unlike transposable elements, which are eliminated from the somatic genome during programmed rearrangements, the PGM gene is found in one copy per haploid genome in the MAC (Aury et al. 2006; Arnaiz et al. 2007). Furthermore, it does not appear to be part of a transposable structure, since no terminal inverted repeats can be found in the sequences surrounding the conserved central region, in the very short upstream and downstream intergenic regions $(91$ and 32 $\mathrm{bp}$, respectively), or up to $900 \mathrm{bp}$ into the flanking genes (data not shown). Altogether, these observations suggest 
A
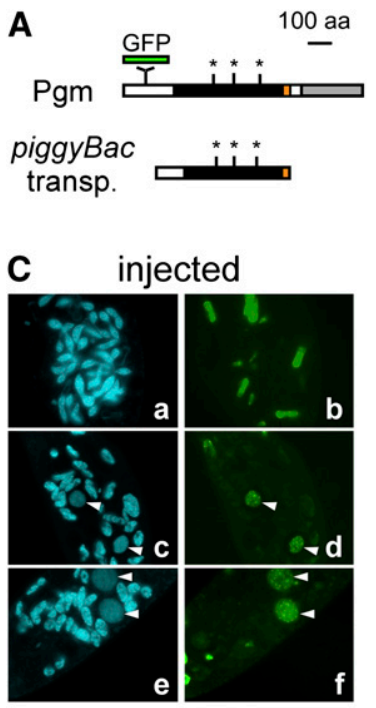

DAPI

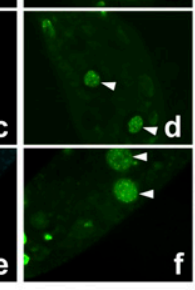

GFP
B

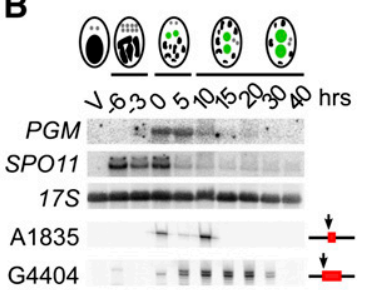

non-injected control

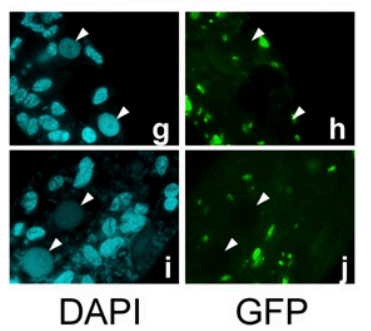

Figure 1. Developmentally regulated expression and cellular localization of the PiggyMac domesticated transposase. $(A)$ Structure of the predicted Pgm protein and comparison with the piggyBac transposase from Trichoplusia ni. The catalytic D residues are indicated by asterisks. (Black boxes) Conserved core. The conserved Cys-rich domain is shown in orange, and the C-terminal coiled-coil domain (predicted by the Lupas algorithm) is shown in gray. ( $B$, top two panels) Northern blot analysis of PGM and PtSPO11 mRNA levels during autogamy of 51new. (Bottom two panels) Ligationmediated PCR (LMPCR) detection of DSBs at the left boundary of IESs 51A1835 and 51G4404 in the same autogamy time course (see Supplemental Fig. S2). (V) Vegetative cells. (C) Localization of the GFP-Pgm fusion in new developing MACs. (Panels $a-f$ ) A vegetative 51new cell was injected with linearized plasmid pPBL49-GFP-M, and autogamy was induced. MAC fragmentation was monitored by DAPI staining (panels $a, c, e, g, i)$, and GFP fluorescence was examined with a Leica microscope (panels $b, d, f, h, j)$. (White arrowheads) Developing new MACs. Note the punctuate nuclear GFP fluorescence pattern in panels $d$ and $f$. Additional fluorescent speckles were sometimes observed with the GFP filter in noninjected cells (panels $h, j)$ and in cells injected with GFP constructs (panel $b$ ), due to the presence of birefringent crystals in the cytoplasm. These do not colocalize with nuclear DAPI fluorescence.

that $P G M$ has evolved through the domestication of a piggyBac transposase gene. In addition, the Pgm protein exhibits an $\sim 300$-amino-acid C-ter extension with a predicted coiled-coil structure not found in any piggyBac transposase (Fig. 1A).

We followed $P G M$ expression during the sexual cycle, which starts with MIC meiosis and proceeds through the development of new MACs. Northern blot hybridization of total RNA with a PGM probe failed to detect any transcript in vegetative cells but showed the specific accumulation of a 3-kb mRNA during autogamy, a selffertilization process (Fig. 1B). Expression of PGM takes place several hours later than expression of the PtSPO11 gene, which encodes the $P$. tetraurelia homolog of the Spo11p endonuclease, essential for meiotic recombination (Keeney 2001). PGM mRNA can be detected when new MACs have started to develop, concomitantly with the detection of the first DSBs at IES ends (Fig. 1B). In addition, expression of a GFP-Pgm fusion showed that the protein localizes preferentially in the developing new MACs during autogamy (Fig. 1C), where programmed

DSBs take place. No detectable fluorescence was observed in vegetative cells (data not shown).

To test the function of the PGM gene, we knocked down its expression by RNAi during autogamy using the "feeding" technique, which consists of feeding Paramecium Escherichia coli bacteria that have been engineered to produce dsRNA from the target gene (Galvani and Sperling 2002). Following silencing of PGM, autogamy started normally, and developing new MACs could be detected by DAPI staining of cells (Supplemental Fig. S3). However, $\sim 95 \%$ of post-autogamous progeny were unable to resume normal vegetative growth when returned to standard growth medium (Table 1). To control for possible off-target silencing artifacts, two nonoverlapping fragments of $P G M$ were used independently to induce RNAi (Fig. 2A), and similar results were obtained with both constructs. For one construct, the efficiency of $P G M$ silencing was checked by Northern blot hybridization of total RNA extracted throughout autogamy from control and PGM silenced cells (Supplemental Fig. S4): No accumulation of full-size mRNA was observed at early time points in cells submitted to RNAi.

Autogamy includes meiosis of the MIC and karyogamy, followed by the development of new MACs: A defect in any of these steps would compromise the recovery of viable progeny. In contrast, conjugation-a sexual process involving reciprocal fertilization between two partnersallows us to distinguish the first set of events, which take place within Paramecium mating pairs, from the development of new MACs, which starts right after pair separation (Berger 1973). Therefore, we used conjugation to identify which steps of the sexual cycle are affected by the silencing of $P G M$. A parallel RNAi experiment was performed for the gene PtSPO11. Reactive cells of both mating types obtained in silencing medium were mixed, and stable mating pairs were transferred to the same medium to monitor exconjugant survival. In agreement with the data obtained during autogamy (Table 1), the silencing of either PtSPO11 or PGM throughout meiosis and subsequent steps of conjugation strongly compromised exconjugant survival (Fig. 2B). In parallel, we crossed reactive cells obtained in standard Klebsiella pneumoniae medium, then transferred the mating pairs to PtSPO11 or PGM silencing medium to allow MIC meiosis and karyogamy to take place normally, while ensuring that exconjugants would start to ingest dsRNA-producing bacteria as soon as they separate (Fig. 2C). Under these conditions, strong lethality was observed only in the PGM knockdown, while the silencing of PtSPO11 had little or no effect, as expected for a meiosis-restricted function. Therefore, $P G M$ gene expression is essential during MAC development for the production of viable sexual progeny.

To gain further insight into the role of Pgm in MAC development, we monitored DNA rearrangements in two independent autogamy time-course experiments, where the PGM gene was silenced by RNAi. In control RNAi experiments (Fig. 3A, left panel), IESs were amplified transiently together with their flanking sequences, before their excision from the new developing MACs: At later time points, only the rearranged forms, originating from both the old and new MACs, could be detected. In contrast, IES-retaining forms (IES ${ }^{+}$) accumulated throughout autogamy in the new MACs of PGM silenced cells, relative to the rearranged sequences present in the fragments of the parental MAC, as visualized by Southern 
Baudry et al.

Table 1. Survival of post-autogamous progeny of P. tetraurelia cells silenced for genes PGM and PtSPO11

\begin{tabular}{|c|c|c|c|c|c|c|}
\hline \multirow{3}{*}{$\begin{array}{l}\text { Medium } \\
\text { Target gene } \\
\text { Silencing plasmid }\end{array}$} & \multicolumn{5}{|c|}{ Silencing } & \multirow{3}{*}{$\begin{array}{c}\text { Standard } \\
\text { None } \\
\text { None }\end{array}$} \\
\hline & \multicolumn{2}{|c|}{$P G M$} & \multicolumn{2}{|c|}{ PtSPO11 } & \multirow{2}{*}{$\begin{array}{l}\text { ND7 } \\
\text { ND7 }\end{array}$} & \\
\hline & PGM-1 & PGM-2 & PtSPO11-1 & PtSPO11-2 & & \\
\hline$\%$ Wild type & 3 & 2 & 6 & 11 & 96 & 98 \\
\hline$\%$ sick & 2 & 6 & 8 & 14 & 0 & 0 \\
\hline$\%$ dead & 95 & 92 & 86 & 75 & 4 & 2 \\
\hline Total \% & 100 & 100 & 100 & 100 & 100 & 100 \\
\hline Cells & 132 & 204 & 144 & 84 & 324 & 222 \\
\hline Number of experiments & (5) & (7) & (5) & (3) & (11) & (7) \\
\hline
\end{tabular}

The gene targeted in each silencing experiment is indicated in italics. For PGM and PtSPO11, the RNAi plasmids used for dsRNA production are displayed (see Fig. 2A). The ND7 gene was used as a control RNAi target, since its silencing has no effect on sexual processes (Nowacki et al. 2005). Autogamy was also performed in standard K. pneumoniae medium (right column). Cells were starved in each medium to induce autogamy and, following 3-4 d of starvation, autogamous cells were transferred individually to $K$. pneumoniae medium to monitor growth of sexual progeny. The table summarizes the data from several independent experiments (see Supplemental Table S2 for details) and shows the total number of autogamous cells analyzed for each RNAi. The number of experiments is indicated in parentheses. (sick) Slow-growing progeny, often larger cells than wild-type and slow-swimming; (dead) death in progeny after less than three cell divisions.

blot hybridization (Fig. 3A; Supplemental Fig. S5A) or by PCR amplification (Supplemental Fig. S6A) of IEScontaining regions. At the latest time points, $\mathrm{IES}^{+}$forms accounted for up to $\sim 30 \%$ of total genomic DNA for each locus, due to overamplification of the nonrearranged region in the developing new MAC and to the degradation of parental MAC fragments that occurs during prolonged starvation (Berger 1974). Because of the presence of the rearranged genome in the old MAC, it is generally difficult to detect new excision junctions in the developing MAC. The variant cell line $51 \Delta A$ carries a maternally inherited MAC deletion of the surface antigen A gene but retains the gene in its MIC. We therefore used this strain to monitor the transient appearance of IES excision junctions within this locus, but failed to detect any in cells depleted for Pgm (Supplemental Fig. S6B). Ligationmediated PCR experiments established a clear correlation between the above defects in IES excision and a complete absence of DSBs at the ends of all IESs tested (Fig. 3B).

In the $51 \Delta A$ cell line, Southern blot hybridization with a probe specific for the $A$ gene region (Fig. 4) clearly revealed the accumulation of a high-molecular-weight species (band 1) representing chromosomes carrying the full-length $A$ gene. Consistent with our previous observations, these chromosomes retain their IESs, as demonstrated by the size difference relative to the normal MAC fragment (band 2) observed in the wild-type 51new strain (Fig. 4, middle panel) and by hybridization with an IESspecific probe (Fig. 4, bottom panel). Thus, like IES excision, maternally inherited elimination of the $A$ gene is blocked in PGM silenced cells, and the nonrearranged germline locus is retained in the developing new MAC. Similar results were obtained for the germline region located downstream from the surface antigen G gene, which contains a transposon that is eliminated imprecisely during MAC development, leading to chromosome fragmentation (Supplemental Fig. S5B,C; data not shown). Again, the silencing of $P G M$ led to retention of the MIC sequences located downstream from the normal telomere addition site.

The essential role of the PGM piggyBac transposase in $P$. tetraurelia genome rearrangements may be an addi- tional example of how capture of transposon-coding sequences by a host genome can allow for the emergence of a new cellular function. A well-known example of such domestication is the $\mathrm{V}(\mathrm{D}) \mid \mathrm{J}$ recombination system of

A

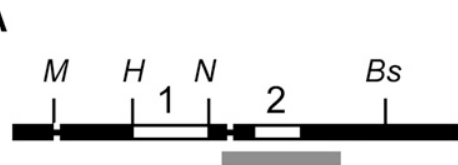

$500 \mathrm{bp}$

PGM
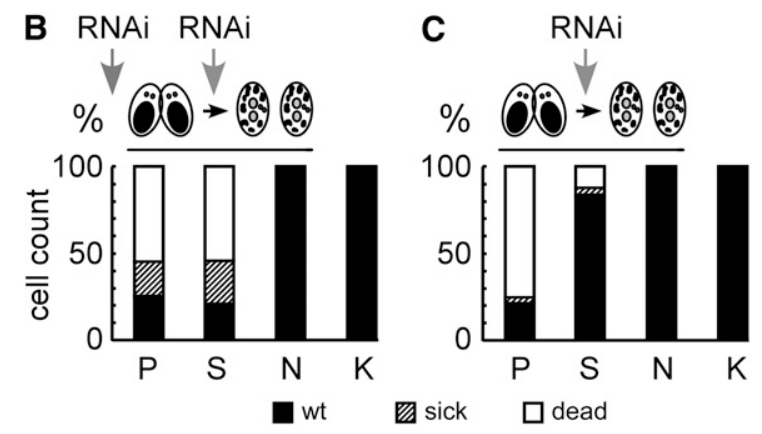

Figure 2. Post-transcriptional silencing of the $P G M$ gene during MAC development. (A) Maps of genes PGM and PtSPO11 showing fragments used as Northern blot hybridization probes (gray line for $P G M$; the whole gene was used for PtSPO11), and for the construction of RNAi plasmids PGM-1 and PGM-2 (1 and 2 on PGM map) and PtSPO11-1 and PtSPO11-2 (1 and 2 on PtSPO11 map). The two $P G M$ introns (thin lines) were confirmed by fine restriction analysis and sequencing of RT-PCR products. Restriction sites are indicated. (M) MscI; (H) HindIII; (N) NcoI; (Bs) BsmBI. (B) PGM silencing during conjugation. 51new $\mathrm{mt} 7$ and $\mathrm{mt} 8$ reactive cells were obtained in induced silencing bacteria harboring RNAi plasmids PGM-2 (P) or PtSPO11-1 (S). The silencing of ND7 (N) was used as a control RNAi. Following conjugation, mating pairs were transferred to each corresponding silencing medium. A conjugation in standard K. pneumoniae medium $(\mathrm{K})$ was also performed. The phenotype of exconjugants is displayed as histograms (see footnote for Table 1). (C) PGM silencing following mating pair separation. Reactive cells were obtained in standard $K$. pneumoniae medium, and mating pairs were transferred to silencing medium. 

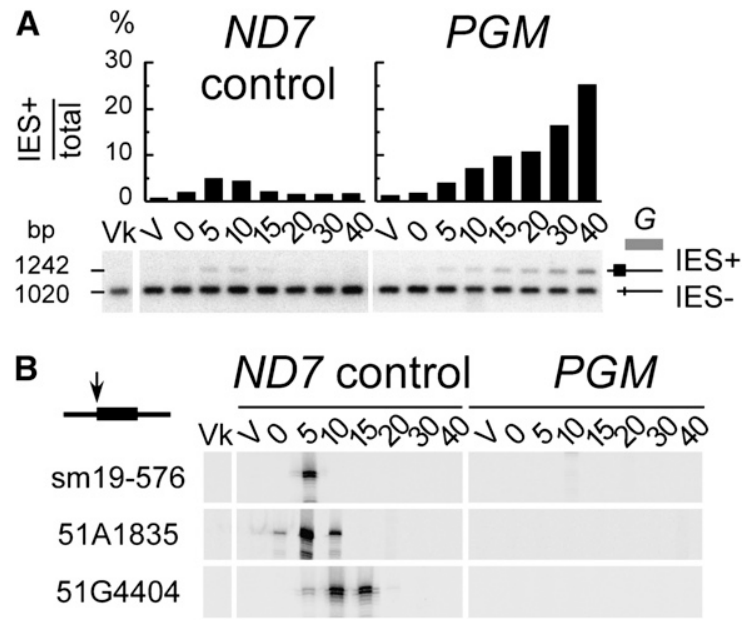

Figure 3. Inhibition of IES 51G4404 excision in PGM silenced $51 \Delta A$ autogamous cells. $(A)$ Quantification of IES-retaining chromosomes $\left(\mathrm{IES}^{+}\right)$on Southern blots of PstI-restricted DNA during autogamy. (Gray box) Hybridization probe Gmac; (Vk) vegetative cells grown in $K$. pneumoniae before transfer to each silencing medium. All time points are in hours (see Supplemental Fig. S4). (B) LMPCR analysis of DSBs at the left arm of each IES excision site in the same time-course experiment.

immunoglobulin genes in vertebrates, which originates from Transib class II transposons (Kapitonov and Jurka 2005). Although this will need to be definitively established by future biochemical studies, we propose that the Pgm domesticated transposase is the endonuclease responsible for IES excision in $P$. tetraurelia because it is essential for IES end cleavage in vivo and it has retained the conserved catalytic site of piggyBac transposases. Interestingly, depletion of this protein also blocks the imprecise elimination of germline DNA that is associated with chromosome fragmentation. This suggests that Pgm-induced DNA DSBs are involved in the two types of programmed DNA elimination described in Paramecium, as has been proposed to explain the common presence of TA dinucleotides in both types of MAC junctions (Le Mouël et al. 2003).

Paramecium IESs are thought to be degenerated copies of ancestral Tc/mariner transposons that would have evolved under selective pressure for precise excision during MAC development. This is somewhat reminiscent of the evolution of nonautonomous miniature transposable clements (MITEs), which have derived from class II transposons through the loss of their coding capacity but still carry conserved terminal inverted repeats (TIRs) at their ends (Wessler et al. 1995; Casacuberta and Santiago 2003). However, IESs and MITEs differ by several aspects. While MITEs can be found in thousands of homogeneous copies in a given genome, each IES is a single-copy element in the haploid genome. In addition, the recognizable TIRs found at MITE ends can be targets for the transposase of related full-length transposons (Feschotte et al. 2005; Loot et al. 2006), whereas IESs do not have canonical TIRs, be they similar to those of their putative Tc/mariner ancestors or to the ends of known piggyBac transposons (Penton et al. 2002). This raises the question of how the Pgm domesticated transposase is targeted to its cleavage sites. It was shown previously that a specialized RNAi pathway mediates trans-nuclear cross-talk between the Paramecium germline and somatic genomes, through a sequence homology-dependent comparison of two noncoding RNA populations produced from the meiotic MIC and the old MAC, respectively (Lepère et al. 2008; 2009). As a result, MIC-specific small RNAs are thought to trigger epigenetic modifications on homologous sequences in the developing new MAC, which could provide a signal for recruitment of the IES excisase. The putative PHD domain of Pgm may participate in the recognition of differentially methylated histones at epigenetically marked sites, as was demonstrated previously for the RAG2 PHD domain, which directs the $\mathrm{V}(\mathrm{D}) \mathrm{J}$ RAG1/2 recombinase to chromatin regions carrying trimethylated $\mathrm{H} 3 \mathrm{~K} 4$ residues (Matthews et al. 2007). The long C-ter extension found in Pgm may also allow protein-protein interactions with other components of the excision machinery.

Close survey of the MAC genome of another ciliate, Tetrahymena thermophila (Eisen et al. 2006), revealed the presence of two genes encoding proteins homologous to Pgm and carrying the conserved DDD catalytic triad (Supplemental Fig. S1). The closest one (TTHERM 01107220) also carries a coiled-coil C-ter extension and is specifically expressed at late stages of conjugation, when germline DNA elimination takes place (Miao et al. 2009). In addition, the developmental DSBs detected at Tetrahymena IES boundaries also exhibit 4-base 5' overhangs, like those observed at Paramecium IES ends, although no conserved TA dinucleotide was reported at DNA cleavage sites in Tetrahymena (Saveliev and Cox 1996). The participation of piggyBac-derived proteins in programmed DNA rearrangements needs to be investigated in Tetrahymena, but their existence suggests that domestication of a piggyBac transposase gene occurred

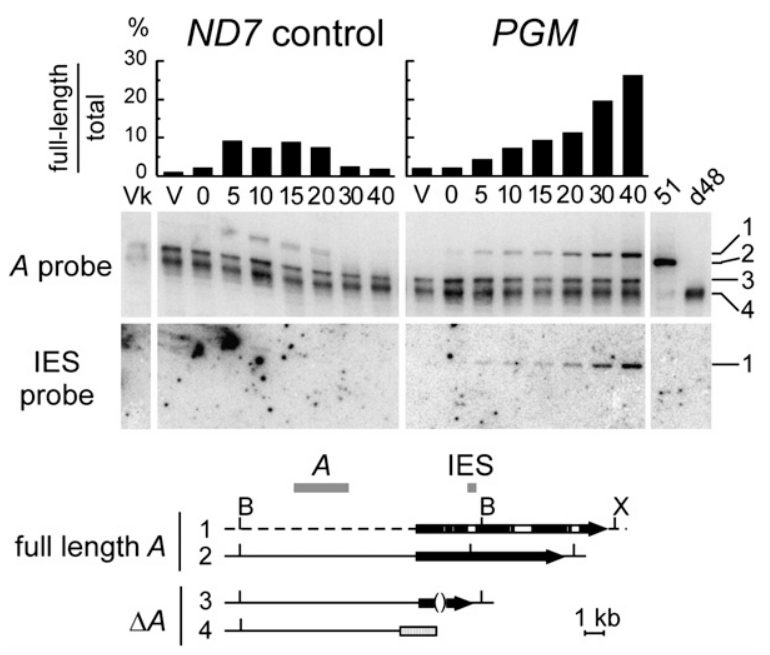

Figure 4. Inhibition of the imprecise deletion of the surface antigen A gene. A Southern blot of BglII-XhoI-restricted genomic DNA was hybridized with probe $A$ (middle panels and histograms on the top), then with IES 51A2591 probe (bottom panels). Species 1 and 2 are absent from the vegetative old MAC (lanes Vk and V) and carry the full-length $A$ gene (1: with IESs; 2 : without IES, as in 51 new). Species 3 is present in the old MAC of the particular clone used here and carries an internal deletion of the $A$ gene. Species 4 is also found in the old MAC and carries a telomere branched upstream of $A$, as in the MAC of the d48 control (a macronuclear variant harboring a telomeric deletion of the $A$ gene) (Forney and Blackburn 1988). 
early during the evolution of oligohymenophorean ciliates. As already suggested by others (Klobutcher and Herrick 1997), Paramecium may have taken advantage of this early domestication to cope with the putative invasion of its germline genome by the Tc/mariner elements that led to present-day TA-IESs. More distant ciliates, such as Oxytricha trifallax, appear to have evolved a different strategy to rearrange their somatic genome and rely on the use of the DDE transposase encoded by their own germline transposons, which do not belong to the piggyBac family (Nowacki et al. 2009). Our work provides the first demonstration of an essential role for a domesticated piggyBac transposase in developmentally programmed DNA rearrangements. Cellular genes encoding proteins related to piggyBac transposases have been reported in many other eukaryotic genomes, and one human gene (PGBD4) carries a potentially active catalytic site (Sarkar et al. 2003). Exploring the function of these genes will certainly be a fascinating avenue for future research.

\section{Materials and methods}

\section{Paramecium strains, cultivation, and autogamy}

All experiments were carried out with strain 51new (Gratias and Bétermier 2003). The $51 \Delta A$ cell line was obtained as described (Gratias et al. 2008), and the absence of the rearranged version of the $A$ gene in the MAC was checked by a PCR assay on total genomic DNA, using primers flanking IESs 51A1835 and 51A4404. Cultivation and autogamy were carried out at $27^{\circ} \mathrm{C}$ as described previously (Gratias and Bétermier 2001). In each timecourse experiment, the $t=0$ time point was defined as the time when $50 \%$ of cells exhibited a fragmented old MAC.

\section{Injection of GFP fusion transgenes}

Plasmids carrying GFP fusion transgenes (see the Supplemental Material) were linearized by AflIII and microinjected into the MAC of vegetative 51new cells, as described (Nowacki et al. 2005). Both C-ter and N-ter fusions gave similar results, and no lethality was observed in the postautogamous progeny of injected cells (data not shown), indicating that the GFP-Pgm fusions did not interfere with normal progression of autogamy (Fig. 1C; data not shown).

\section{Gene silencing experiments}

Preparation of silencing medium. All dsRNA-producing plasmids (see the Supplemental Material) were transformed into E. coli HT115 (Timmons et al. 2001). Silencing media were prepared basically as described in Galvani and Sperling (2002), by inoculating precultures of the appropriate bacterial strains into WGP medium containing $0.1 \mathrm{mg} / \mathrm{mL}$ ampicillin to a starting $\mathrm{OD}_{600}=0.35-0.45$. Following $6-8 \mathrm{~h}$ of shaking at $37^{\circ} \mathrm{C}$, bacterial cultures were diluted sixfold into the same medium containing $0.4 \mathrm{mM}$ IPTG to induce the synthesis of dsRNA. Following overnight induction at $37^{\circ} \mathrm{C}$, silencing media were supplemented with $0.8 \mu \mathrm{g} / \mathrm{mL} \beta$-sitosterol (Merck) before use.

RNAi during autogamy. P. tetraurelia cells were grown in standard $K$. pneumoniae medium for 20-30 vegetative fissions then washed twice in silencing medium. Cells were grown for eight to 10 additional vegetative fissions ( $2 \mathrm{~d}$ ) in silencing medium (freshly induced medium was added the second day) before starvation-induced autogamy. Progression of autogamy was monitored by DAPI staining, and cells were generally $100 \%$ autogamous at day 1 of starvation. At day 3 or 4, 24-30 autogamous cells were picked and transferred individually to $200 \mu \mathrm{L}$ of $K$. pneumoniae medium to monitor growth of sexual progeny.

RNAi during conjugation. P. tetraurelia reactive cells of both mating types were obtained in either $K$. pneumoniae or silencing medium. Following mixing, they were incubated for $1 \mathrm{~h} 45 \mathrm{~min}$ at room temper- ature to allow the first conjugating pairs to form. Freshly induced silencing medium $(2 \mathrm{vol})$ was added, and cells were incubated for 45 min at $27^{\circ} \mathrm{C}$ to stop further conjugation. In each experiment, 12 mating pairs were picked and transferred individually into silencing medium to monitor growth of exconjugants.

\section{Northern and Southern blot hybridization}

Total RNA or genomic DNA was extracted from 200-400 mL of Paramecium cultures during vegetative growth (500-1000 cells per milliliter) or at different time points during an autogamy time course. For RNA extraction, we used the Trizol procedure (Invitrogen) modified by the addition of glass beads to lyse cells. Twenty micrograms of denatured total RNA were separated on a $1 \%$ agarose gel in Tris Borate EDTA, transferred to Hybond $\mathrm{N}^{+}$membrane in $20 \times$ SSC buffer, and UV-cross-linked. Total genomic DNA extraction was performed as described /Gratias and Bétermier 2003). Following restriction enzyme digestion, $2 \mu \mathrm{g}$ of total genomic DNA (quantified using the Invitrogen QuBit assay kit) were electrophoresed on $0.8 \%$ (in Tris Acetate EDTA) or $1 \%$ (in Tris Borate EDTA) agarose gels and transferred to Hybond $\mathrm{N}^{+}$membranes in $0.4 \mathrm{~N}$ $\mathrm{NaOH}$.

Double-stranded probes were labeled by random priming with $\left[\alpha-{ }^{32} \mathrm{P}\right] \mathrm{dATP}$ (3000 Ci/mmol; NEN Radiochemicals, Perkin Elmer), using the Prime-a-gene kit (Promega). Oligonucleotide probes were labeled with $\left[\gamma_{-}{ }^{32} \mathrm{P}\right]$ ATP (3000 Ci/mmol; NEN Radiochemicals, Perkin Elmer) and T4 polynucleotide kinase (Fermentas). Northern blots were hybridized at $42^{\circ} \mathrm{C}$ in Ultrahyb buffer (Ambion) and washed as recommended by the supplier. Southern blots were hybridized at $60^{\circ} \mathrm{C}$ as described (Bétermier et al. 2000) and washed in $0.2 \times$ SSC and $0.1 \%$ SDS at $60^{\circ} \mathrm{C}$ prior to image plate exposure. Plates were scanned with a Typhoon scanner (GE Healthcare Life Sciences). All details about PCR probes can be found in Supplemental Table S1. Probe $A$ (referred to as probe $\mathrm{d}$ in Garnier et al. 2004 ) is an $\sim 3$-kb EcoRI MAC fragment located $\sim 5 \mathrm{~kb}$ upstream of the $A$ gene.

\section{Detection of DSBs by ligation-mediated PCR (LMPCR)}

For the detection of broken chromosome ends, LMPCR was performed as described in Gratias et al. (2008). Briefly, linkers I'/(ATAA)J', I'/(GTAT)J', and $\mathrm{I}^{\prime} /(\mathrm{ATAC}) \mathrm{J}^{\prime}$ were ligated to broken chromosome ends at the left boundary of IESs 51A1835 (from the surface antigen A gene), 51G4404 (from the surface antigen G gene), and sm19-576 (from the $\eta$ tubulin SM19 gene), respectively. Primer "PCR haut" was used in combination with a specific primer for the amplification of each ligation product. Following primer extension from a ${ }^{32} \mathrm{P}$-labeled nested specific primer, LMPCR products were visualized on $6 \%$ polyacrylamide and $7 \mathrm{M}$ urea sequencing gels. Primer and linker sequences can be found in Gratias et al. (2008).

\section{Acknowledgments}

We thank the students of the 2007 Pasteur course on "Genome Analysis" who performed preliminary RNAi experiments related to this study. We are grateful to all members of the Bétermier and Meyer laboratories for stimulating discussions; to Julien Bischerour, Bénédicte Michel, and Linda Sperling for critical reading of the manuscript; and to Bernard Hallet for suggesting a name for the Paramecium domesticated transposase. This work was supported by a CNRS ATIP grant to M.B., and by ANR grants (\#NT05_1522 and \#ANR-08-BLAN-0233) to E.M. and M.B. A.K. is supported by a PhD fellowship from the MESR.

\section{References}

Arnaiz O, Cain S, Cohen J, Sperling L. 2007. ParameciumDB: A community resource that integrates the Paramecium tetraurelia genome sequence with genetic data. Nucleic Acids Res 35: D439D444. doi: 10.1093/nar/gk1777.

Aury JM, Jaillon O, Duret L, Noel B, Jubin C, Porcel BM, Segurens B, Daubin V, Anthouard V, Aiach N, et al. 2006. Global trends of wholegenome duplications revealed by the ciliate Paramecium tetraurelia. Nature 444: 171-178.

Berger JD. 1973. Nuclear differentiation and nucleic acid synthesis in wellfed exconjugants of Paramecium aurelia. Chromosoma 42: 247-268. 
Berger JD. 1974. Selective autolysis of nuclei as a source of DNA precursors in Paramecium aurelia exconjugants. I Protozool 21: 145-152.

Bétermier M. 2004. Large-scale genome remodelling by the developmentally programmed elimination of germ line sequences in the ciliate Paramecium. Res Microbiol 155: 399-408.

Bétermier M, Duharcourt S, Seitz H, Meyer E. 2000. Timing of developmentally programmed excision and circularization of Paramecium internal eliminated sequences. Mol Cell Biol 20: 1553-1561.

Casacuberta JM, Santiago N. 2003. Plant LTR-retrotransposons and MITEs: Control of transposition and impact on the evolution of plant genes and genomes. Gene 311: 1-11.

Eisen JA, Coyne RS, Wu M, Wu D, Thiagarajan M, Wortman JR, Badger $\mathrm{JH}$, Ren Q, Amedeo P, Jones KM, et al. 2006. Macronuclear genome sequence of the ciliate Tetrahymena thermophila, a model eukaryote. PLoS Biol 4: e286. doi: 10.1371/journal.pbio.0040286.

Elick TA, Bauser CA, Fraser MJ. 1996. Excision of the piggyBac transposable element in vitro is a precise event that is enhanced by the expression of its encoded transposase. Genetica 98: 33-41.

Feschotte C, Osterlund MT, Peeler R, Wessler SR. 2005. DNA-binding specificity of rice mariner-like transposases and interactions with Stowaway MITEs. Nucleic Acids Res 33: 2153-2165.

Forney JD, Blackburn EH. 1988. Developmentally controlled telomere addition in wild-type and mutant paramecia. Mol Cell Biol 8: 251258.

Galvani A, Sperling L. 2002. RNA interference by feeding in Paramecium. Trends Genet 18: 11-12.

Garnier O, Serrano V, Duharcourt S, Meyer E. 2004. RNA-mediated programming of developmental genome rearrangements in Paramecium tetraurelia. Mol Cell Biol 24: 7370-7379.

Gratias A, Bétermier M. 2001. Developmentally programmed excision of internal DNA sequences in Paramecium aurelia. Biochimie 83: 1009-1022.

Gratias A, Bétermier M. 2003. Processing of double-strand breaks is involved in the precise excision of Paramecium IESs. Mol Cell Biol 23: 7152-7162.

Gratias A, Lepere G, Garnier O, Rosa S, Duharcourt S, Malinsky S, Meyer E, Betermier M. 2008. Developmentally programmed DNA splicing in Paramecium reveals short-distance crosstalk between DNA cleavage sites. Nucleic Acids Res 36: 3244-3251.

Jahn CL, Klobutcher LA. 2002. Genome remodeling in ciliated protozoa. Annu Rev Microbiol 56: 489-520.

Kapitonov VV, Jurka J. 2005. RAG1 core and V(D)J recombination signal sequences were derived from Transib transposons. PLOS Biol 3: e181. doi: 10.1371/journal.pbio.0030181.

Keeney S. 2001. Mechanism and control of meiotic recombination initiation. Curr Top Dev Biol 52: 1-53.

Klobutcher LA, Herrick G. 1995. Consensus inverted terminal repeat sequence of Paramecium IESs: Resemblance to termini of Tc1-related and Euplotes Tec transposons. Nucleic Acids Res 23: 2006-2013.

Klobutcher LA, Herrick G. 1997. Developmental genome reorganization in ciliated protozoa: The transposon link. Prog Nucleic Acid Res Mol Biol 56: 1-62.

Le Mouël A, Butler A, Caron F, Meyer E. 2003. Developmentally regulated chromosome fragmentation linked to imprecise elimination of repeated sequences in Paramecium. Eukaryot Cell 2: 1076 1090.

Lepère G, Bétermier M, Meyer E, Duharcourt S. 2008. Maternal noncoding transcripts antagonize the targeting of DNA elimination by scanRNAs in Paramecium tetraurelia. Genes \& Dev 22: 1501-1512.

Lepère G, Nowacki M, Serrano V, Gout JF, Guglielmi G, Duharcourt S, Meyer E. 2009. Silencing-associated and meiosis-specific small RNA pathways in Paramecium tetraurelia. Nucleic Acids Res 37: 903-915

Loot C, Santiago N, Sanz A, Casacuberta JM. 2006. The proteins encoded by the pogo-like Lemil element bind the TIRs and subterminal repeated motifs of the Arabidopsis Emigrant MITE: Consequences for the transposition mechanism of MITEs. Nucleic Acids Res 34: $5238-5246$

Matthews AG, Kuo AJ, Ramon-Maiques S, Han S, Champagne KS, Ivanov D, Gallardo M, Carney D, Cheung P, Ciccone DN, et al. 2007. RAG2 $\mathrm{PHD}$ finger couples histone $\mathrm{H} 3$ lysine 4 trimethylation with $\mathrm{V}(\mathrm{D}) \mathrm{J}$ recombination. Nature 450: 1106-1110.
Miao W, Xiong J, Bowen J, Wang W, Liu Y, Braguinets O, Grigull J, Pearlman RE, Orias E, Gorovsky MA. 2009. Microarray analyses of gene expression during the Tetrahymena thermophila life cycle. PLoS ONE 4: e4429. doi: 10.1371/journal.pone.0004429.

Mitra R, Fain-Thornton J, Craig NL. 2008. piggyBac can bypass DNA synthesis during cut and paste transposition. EMBO J 27: 1097-1109.

Nowacki M, Zagorski-Ostoja W, Meyer E. 2005. Nowalp and Nowa2p: Novel putative RNA binding proteins involved in trans-nuclear crosstalk in Paramecium tetraurelia. Curr Biol 15: 1616-1628.

Nowacki M, Higgins BP, Maquilan GM, Swart EC, Doak TG, Landweber LF. 2009. A functional role for transposases in a large eukaryotic genome. Science 324: 935-938.

Penton EH, Sullender BW, Crease TJ. 2002. Pokey, a new DNA transposon in Daphnia (Cladocera: Crustacea). J Mol Evol 55: 664-673.

Plasterk RHA, van Luenen HGAM. 2002. The Tc1/mariner family of transposable elements. In Mobile DNA II (eds. N Craig et al.), pp. 519-532. American Society for Microbiology, Washington, DC.

Sarkar A, Sim C, Hong YS, Hogan JR, Fraser MJ, Robertson HM, Collins FH. 2003. Molecular evolutionary analysis of the widespread piggyBac transposon family and related 'domesticated' sequences. Mol Genet Genomics 270: 173-180.

Saveliev SV, Cox MM. 1996. Developmentally programmed DNA deletion in Tetrahymena thermophila by a transposition-like reaction pathway. EMBO I 15: 2858-2869.

Timmons L, Court DL, Fire A. 2001. Ingestion of bacterially expressed dsRNAs can produce specific and potent genetic interference in Caenorhabditis elegans. Gene 263: 103-112.

Wessler SR, Bureau TE, White SE. 1995. LTR-retrotransposons and MITEs: Important players in the evolution of plant genomes. Curr Opin Genet Dev 5: 814-821.

Yao MC, Duharcourt S, Chalker DL. 2002. Genome-wide rearrangements of DNA in ciliates. In Mobile DNA II (eds. NL Craig et al.), pp. 730 758. ASM Press, Washington, DC 


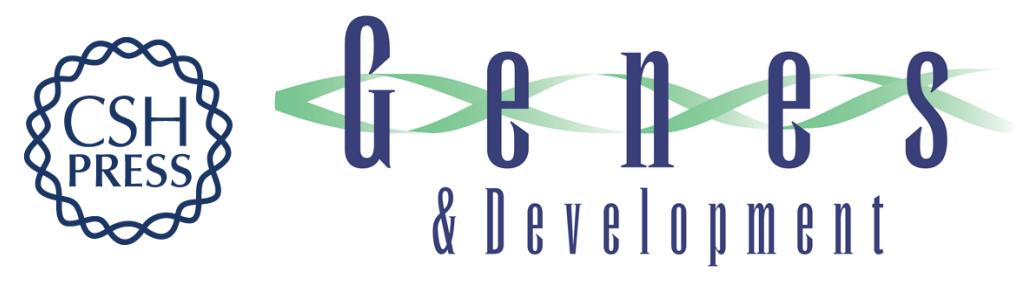

\section{PiggyMac, a domesticated piggyBac transposase involved in programmed genome rearrangements in the ciliate Paramecium tetraurelia}

Céline Baudry, Sophie Malinsky, Matthieu Restituito, et al.

Genes Dev. 2009, 23:

Access the most recent version at doi:10.1101/gad.547309

\section{Supplemental http://genesdev.cshlp.org/content/suppl/2009/11/03/23.21.2478.DC1 \\ Material}

Related Content

Subtraction by addition: domesticated transposases in programmed DNA elimination

Jason A. Motl and Douglas L. Chalker

Genes Dev. November, 2009 23: 2455-2460

References

This article cites 35 articles, 7 of which can be accessed free at:

http://genesdev.cshlp.org/content/23/21/2478.full.html\#ref-list-1

Articles cited in:

http://genesdev.cshlp.org/content/23/21/2478.full.html\#related-urls

\section{License}

Email Alerting

Service

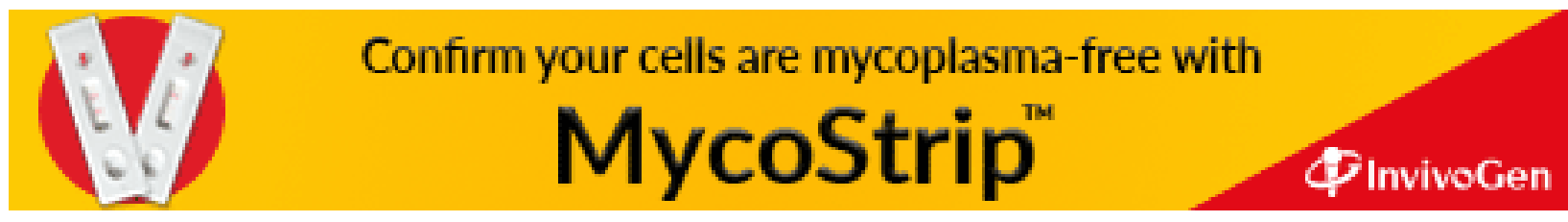

